\title{
Lower Mantle Electrical Conductivity Inferred from Probabilistic Tomography
}

\author{
Frédéric Deschamps \\ Institute of Earth Sciences, Academia Sinica, Taipei, Taiwan
}

Received 14 June 2013, revised 8 April 2014, accepted 19 August 2014

\begin{abstract}
Electrical conductivity is a potentially powerful observable that may be used in combination with seismological models to investigate the 3D thermo-chemical structure of the Earth's mantle because it depends upon both temperature and compositional parameters. Despite strong uncertainties in the lower mantle mineral conductive properties and ambiguities in choosing an appropriate average scheme to estimate the aggregate conductivity from the individual conductivities of each mineral, it is possible to calculate a radial reference model and 3D maps of electrical conductivity in the lower mantle from thermo-chemical reference models and 3D maps. This work presents a first attempt to build such models using probabilistic tomography as the input thermo-chemical structure. The radial model increases from about $0.6 \mathrm{~S} \mathrm{~m}^{-1}$ at the top of the lower mantle to $16 \mathrm{~S} \mathrm{~m}^{-1}$ at its bottom. The 3D structure shows strong lateral variations at both the top $(670-1200 \mathrm{~km})$ and bottom $(2000-2891 \mathrm{~km})$ of the lower mantle, with moderate variations in the 1200 - $2000 \mathrm{~km}$ layer. In the lowermost mantle $(>2000 \mathrm{~km})$, which is not yet imaged yet by electrical conductivity tomographic models, a belt of high conductivity extends along the equator with relative anomalies up to $90 \%$ compared to the horizontal average.
\end{abstract}

Key words: Lower mantle, Thermo-chemical structure, Electrical conductivity

Citation: Deschamps, F., 2015: Lower mantle electrical conductivity inferred from probabilistic tomography. Terr. Atmos. Ocean. Sci., 26, 27-40, doi: 10.3319/TAO.2014.08.19.03(GRT)

\section{INTRODUCTION}

Seismic observations provide the most detailed information so far about the structure of the Earth's lower mantle. Seismic tomography models map relative anomalies in shear and compressional seismic velocity with the most recent global models resolving structures about $500 \mathrm{~km}$ across (e.g., Houser et al. 2008; Kustowski et al. 2008; Ritsema et al. 2011). Models obtained using different datasets and inversion methods now agree on the large scale structure (for a comparison between recent models, see Ritsema et al. 2011). A major, unsolved, issue is to interpret these models in terms of mantle thermo-chemical structure. Seismic velocity anomalies reflect variations in density and elastic moduli (bulk and shear), which in turn result from variations in temperature and composition. Given the pressure, temperature and composition (either petrological or mineralogical), seismic velocity and their 3D variations can be modeled using an appropriate equation of state modeling

\footnotetext{
* Corresponding author

E-mail:frederic@earth.sinica.edu.tw
}

and mineral physics data (e.g., Trampert et al. 2001; Connolly 2005). Because temperature and composition strongly trade-off, seismic velocity anomalies alone cannot resolve the 3D thermo-chemical structure of the mantle. Independent constraints on the density structure, in particular from seismic normal modes (Ishii and Tromp 1999; Trampert et al. 2004; Mosca et al. 2012), help break this trade-off, but do not lift up all ambiguities.

In contrast to density and seismic velocities, the electrical conductivity of mantle materials increases with temperature (e.g., Poirier 1991). It also varies with the mineralogical composition. In particular, magnesio-wüstite is more conductive than perovskite throughout the lower mantle (Vacher and Verhoeven 2007; Khan and Shankland 2012). Therefore, a good knowledge of lateral variations in electrical conductivity potentially provides additional and independent constraints on the mantle thermo-chemical structure. The Earth's magnetic field induces electrical currents in the mantle and crust. These currents depend on the $3 \mathrm{D}$ variations in electrical conductivity and induce small variations in the 
observed magnetic field. Geomagnetic variation inversions with periods from a few days to 1 year, measured at both magnetic observatories and from satellite data, can be used to recover 1D radial profiles and 3D maps of mantle electrical conductivity (Banks 1969; for a detailed treatment, see Kuvshinov and Semenov 2012). Several 1D radial models have been inferred (Olsen 1999; Kuvshinov and Olsen 2006; Velímský 2010), showing an increase in 2 orders of magnitude between the top of the mantle and $1500 \mathrm{~km}$ depth. In the past few years the first regional and global tomographic models of mantle electrical conductivity have been proposed (Kelbert et al. 2009; Shimizu et al. 2010; Tarits and Mandéa 2010; Semenov and Kuvshinov 2012). However, due to data availability and ambiguity concerning the source of long period (1 year and more) magnetic field variations, conductivity at depths greater than $\sim 1600 \mathrm{~km}$ cannot yet be resolved.

Linking the mantle thermo-chemical and electrical conductivity structures further requires mineral physics data and modeling. Electrical conductivities and their sensitivities to temperature and pressure have been measured for the main minerals in the upper and lower mantle (for a recent compilation, see Khan and Shankland 2012). Available data for perovskite ( $\mathrm{Li}$ and Jeanloz 1991; Poirier and Peyronneau 1992; Shankland et al. 1993; Katsura et al. 1998) and magnesio-wüstite (Dobson and Brodholt 2000) were further used to infer 1D radial models of lower mantle conductivity from prescribed 1D radial temperature and composition models (e.g., Xu et al. 2000; Vacher and Verhoeven 2007; Khan and Shankland 2012). So far, however, no 3D model has been proposed.

The purpose of this article is to calculate 3D models of lower mantle electrical conductivity from its thermo-chemical structure using an appropriate electrical conductivity modeling at mantle temperature and pressure (Vacher and Verhoeven 2007).

\section{LOWER MANTLE SEISMIC AND THERMO-CHEMICAL STRUCTURE}

Global models of seismic tomography using different datasets and methods now provide coherent maps of seismic velocity variations in the lower mantle. The strongest heterogeneities are found in the bottom part of the lower mantle, starting around $2400 \mathrm{~km}$ depth and down to the core-mantle boundary (CMB). At these depths the seismic structure of the mantle is dominated by two large low shearwave velocity provinces (LLSVP) beneath the Pacific and Africa, where shear-wave velocity drops by a few percent compared to its horizontally averaged value. By contrast, the mid-lower mantle, between 1000 and $2000 \mathrm{~km}$ is seismically homogeneous at large and medium scales. The RMS of seismic velocity anomalies is small, and no significant pattern appears. The upper part of the lower mantle, between $670-1000 \mathrm{~km}$ depth is moderately heterogeneous, with global tomographic models showing a secondary peak in the radial RMS of shear-wave velocity anomalies. Seismic heterogeneities at these depths may be related to the stacking of subducted slabs (Fukao et al. 2001).

At each depth, lateral seismic velocity anomalies result from lateral variations in temperature and composition. Comparisons between the shear- and compressional-wave velocities anomaly distributions gives first order hints about the nature of these anomalies (van der Hilst and Kárason 1999; Masters et al. 2000). Due to a trade-off between thermal and compositional terms, however, the detailed distributions of these anomalies cannot be resolved using seismic velocity alone. Additional constraints on density allow breaking this trade-off. For instance, probabilistic tomography, which is based on normal modes data providing full probability density functions for the shear-wave velocity, bulk-sound velocity, and density at each point in the model, have been used to determine full distributions of temperature, fractions of silicate [parameterized as $(\mathrm{Mg}, \mathrm{Fe})$-perovskite] and iron oxide (the latter being distributed in perovskite and magnesio-wüstite) (Trampert et al. 2004). Due to normal mode availability and computational resources, the thermo-chemical structure is limited to spherical harmonic degrees 2,4 , and 6 , and to three layers in the lower mantle. These distributions are however robust and indicate that variations in iron and silicate are present throughout the lower mantle, in particular in its lowermost part where LLSVP appears enriched in iron and perovskite (Fig. 1). Interestingly, the thermo-chemical distributions inferred by Trampert et al. (2004) suggest that density variations are a good proxy for variations in iron, whereas bulk-sound velocity anomalies map variations in the fraction of perovskite with a good approximation. By contrast, temperature anomalies cannot be inferred straightforwardly from shear-wave velocity anomalies, as often assumed. Mosca et al. (2012) recently obtained an updated probabilistic tomography model with finer vertical resolution, from which they infer thermo-chemical distributions, including for the postperovskite phase. Interestingly, these thermo-chemical distributions do not substantially differ from those of Trampert et al. (2004). In particular, the large scale structures identified by Mosca et al. (2012) are similar to those in Trampert et al. (2004), and in both models LLSVP appears enriched in iron and perovskite by about 3 and 10\%, respectively.

The choice to parameterize the compositional anomalies in terms of variations in iron and silicate, as in Trampert et al. (2004) and Mosca et al. (2012) may appear somewhat arbitrary. It is however consistent with the hypothesis that lower mantle chemical heterogeneities result from an early chemical differentiation in the mantle (Lee et al. 2010; Nomura et al. 2011). It further agrees with E-chondritic models of Earth's composition (Javoy et al. 2010). Another potential source of chemical heterogeneities in the deep mantle is high-pressure MORB entrained in the deep mantle by subducted slab (e.g., van der Hilst et al. 1997). While patches 
of high-pressure MORB are certainly present in the deep mantle, it has been shown that they would hardly explain the LLSVP (Deschamps et al. 2012).

The thermo-chemical distribution from Trampert et al. (2004), rather than those from Mosca et al. (2012) will be used in this study in combination with mineral physics data (see section 3) to calculate the electrical conductivity distribution. This choice is motivated by the fact that electrical conductivity data for post-perovskite are still sparse.

\section{MODELING LOWER MANTLE ELECTRICAL CONDUCTIVITY}

At mantle pressure and temperature, mantle minerals behave as semi-conductors (e.g., Poirier 1991; Goddat et al. 1999). In iron-bearing hydrous silicates, three main mechanisms, involving different charge-carriers (ionic, small polaron, and proton), contribute to mantle rocks electrical conductivity (e.g., Yoshino et al. 2009). Ionic conduction involves migration of $\mathrm{Mg}$-site vacancies, whereas small polaron conduction results from charge hopping from $\mathrm{Fe}^{3+}-\mathrm{Fe}^{2+}$ ions, while proton conduction is due to proton hopping amongst point defects. In each case charge migration is a diffusion process that can be modeled using the Nernst-Einstein equation (e.g., Poirier 1991), in which the charge carrier concentration is thermally controlled following a Boltzmann distribution. Overall the electrical conductivity associated with each mechanism may be parameterized using an Arrhenius law,

$\sigma=\sigma_{0} T^{m} \exp \left(-\frac{E_{a}+P V_{a}}{k T}\right)$ where $T$ is the temperature, $P$ the pressure, $k=8.617 \times 10^{-5}$ $\mathrm{eV} \mathrm{K}^{-1}$ the Boltzmann constant and $m$ a constant whose theoretical value depends on the type of polaron (Goddat et al. 1999). The values of the pre-exponential factor $\sigma_{0}$, and of the activation energy and activation volume, $E_{a}$ and $V_{a}$, depends on the transport mechanism. The total electrical conductivity is the sum of the Arrhenius law for each conduction mechanism considered. In practice, however, the lower mantle conductivity can be modeled with one single mechanism with a good approximation. Goddat et al. (1999) pointed out that the dominant conduction mechanism in the lower mantle should be small polaron conduction due to the fact that the lower mantle mineral activation energy for this mechanism is small, typically a few tenths of $\mathrm{eV}$ (Table 1). By contrast the activation energy for ionic conduction is larger than $1 \mathrm{eV}$. In addition, proton conduction strongly depends on the water content (Yoshino et al. 2009), and may thus be inefficient in the lower mantle. For perovskite, Vacher and Verhoeven (2007) further pointed out that the sum the two conduction mechanisms (ionic and small polaron) identified by $\mathrm{Xu}$ and McCammon (2002) may be parameterized with a single Arrhenius law, as defined by Xu et al. (2000). They also noted that the influence of the iron content is much more sensitive than accounting for mechanisms with different temperaturedependence. Finally, it is worth noting that for magnesiowüstite, Dobson and Brodholt (2000) identified only one regime for temperatures larger than $1000 \mathrm{~K}$, i.e., at temperatures relevant to the lower mantle.

\subsection{Model and Data}

The electrical conductivity of the lower mantle is

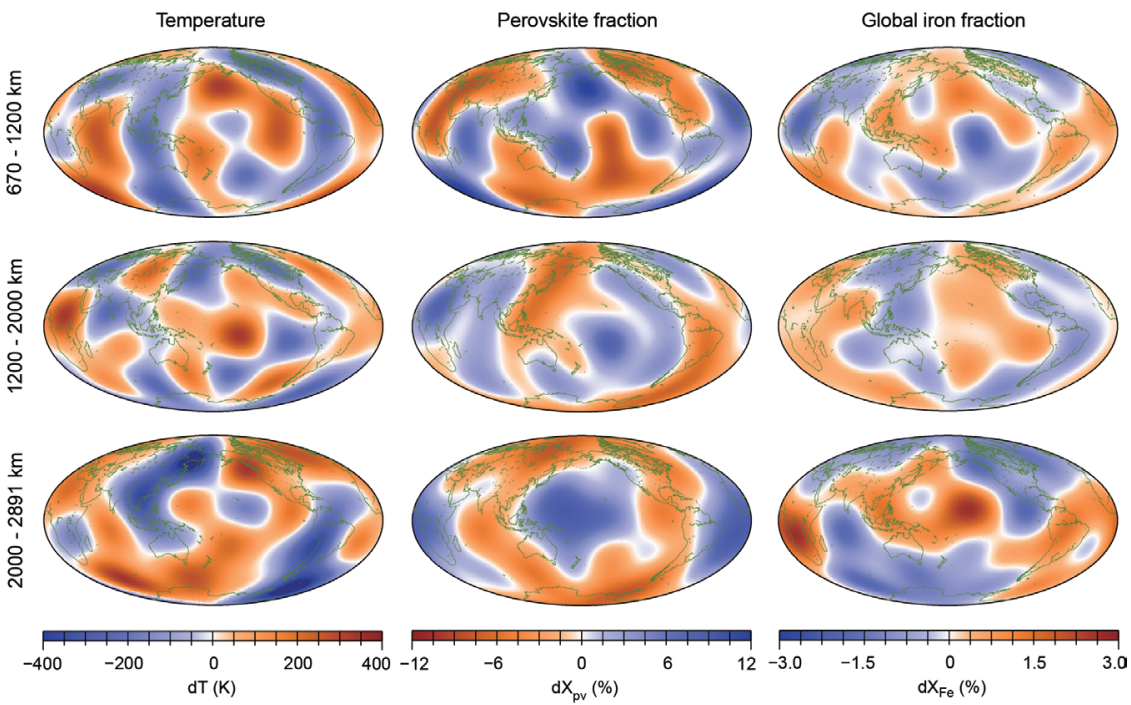

Fig. 1. Thermo-chemical structure of the lower mantle inferred from probabilistic tomography (Trampert et al. 2004). Left, middle, and right column plots anomalies in temperature, fraction of ( $\mathrm{Mg}, \mathrm{Fe})$-perovskite, and global fraction of iron (the iron being distributed in perovskite and magnesiowüstite). Vertical parameterization includes three layers, from top to bottom 670 - 1200, 1200 - 2000, and 2000 - $2891 \mathrm{~km}$. Distributions include spherical harmonic degrees 2,4 , and 6 , and are smoothed on a $1^{\circ} \times 1^{\circ}$ grid. 
Table 1. Electrical conductivity properties of perovskite and magnesio-wüstite.

\begin{tabular}{ccc}
\hline Parameter & Perovskite & Magnesio-wüstite \\
\hline $\log \left(\sigma_{0}\right)$ & $1.28 \pm 0.18$ & $2.56 \pm 0.10$ \\
$E_{a}\left(\mathrm{eV} \mathrm{K}^{-1}\right)$ & $0.68 \pm 0.04$ & $0.88 \pm 0.03$ \\
$V_{a}\left(\mathrm{~cm}^{3} \mathrm{~mol}^{-1}\right)$ & $-0.26 \pm 0.03$ & $-0.26 \pm 0.07$ \\
$\alpha$ & $3.56 \pm 1.32$ & $3.14 \pm 0.07$ \\
$\beta$ & $-1.72 \pm 0.38$ & 0 \\
\hline
\end{tabular}

Note: Listed values were deduced by Vacher and Verhoeven (2007) from experimental data on perovskite (Poirier and Peyronneau 1992; Shankland et al. 1993) and magnesio-wüstite (Dobson and Brodholt 2000). The reference value of the iron fraction is $x_{\text {ref }}=0.1$ for both perovskite and magnesio-wüstite.

modeled following the approach described in Vacher and Verhoeven (2007). Two minerals are considered, perovskite and magnesio-wüstite, and for each of these two minerals the influence of the iron content is taken into account. The individual conductivity $\sigma_{i}$ of each mineral $i$ depends on the temperature $T$, pressure $P$, and individual iron fraction $x_{F e}^{i}$, as follows

$\sigma_{i}=\sigma_{0}^{i}\left(\frac{x_{F e}^{i}}{x_{r e f}^{i}}\right)^{\alpha} \exp \left[-\frac{E_{a}^{i}+\beta\left(x_{F e}^{i}-x_{r e f}^{i}\right)+P V_{a}^{i}}{k T}\right]$

where $\sigma_{0}^{i}$ and $E_{a}^{i}$ are the pre-exponential factor and activation energy of mineral $i$, respectively, calculated at the reference iron fraction $x_{r e f}^{i}$. The values of $x_{r e f}^{i}$ can be arbitrarily chosen, provided that the corresponding input values of $\sigma_{0}^{i}$ and $E_{a}^{i}$ are corrected to match the mineral physics data. Here, $x_{r e f}^{i}$ is set to 0.1 for both perovskite and magnesio-wüstite. For each mineral the sensitivity of conductivity to iron is controlled using two parameters, $\alpha$ and $\beta$, which are deduced from experimental data. The parameters used in this study are similar to those in Vacher and Verhoeven (2007) and are listed in Table 1. These values were deduced from mineral physics experiments on perovskite (Poirier and Peyronneau 1992; Shankland et al. 1993) and on magnesio-wüstite (Dobson and Brodholt 2000). Note that Vacher and Verhoeven (2007) recalculated the pre-exponential factor and activation energy of magnesio-wüstite from the original data from Dobson and Brodholt (2000) in order to cancel the pre-exponential dependence on temperature, i.e., they set $m=0$ in Eq. (1), the original value determined by Dobson and Brodholt (2000) being $m=-0.24$. The iron content in each mineral is controlled by prescribing the global fraction of iron in the assemblage, $X_{F e}$, and the iron partitioning between perovskite and magnesiowüstite, $K_{F e}$, which is defined as

$K_{F e}=\frac{x_{F e}^{p v} /\left(1-x_{F e}^{p v}\right)}{x_{F e}^{m w} /\left(1-x_{F e}^{m w}\right)}$ where $x_{F e}^{p v}$ and $x_{F e}^{m w}$ are the individual fractions of iron in perovskite and magnesio-wüstite, respectively. These individual fractions are further related to the global iron fraction by

$X_{F e}=X_{p v} x_{F e}^{p v}+\left(1-X_{p v}\right) x_{F e}^{m w}$

where $X_{p v}$ is the volume fraction of perovskite in the assemblage. Individual iron fractions are obtained by solving Eqs. (3) and (4). For instance, taking $X_{p v}=0.8, X_{F e}=0.1$, and $K_{F e}=0.5$ leads to $x_{F e}^{p v}=0.086$ and $x_{F e}^{m w}=0.158$. For $K_{F e}=1.0, x_{F e}^{p v}$ and $x_{F e}^{m w}$ are both equal to $X_{F e}$.

The conductivity of the lower mantle assemblage is then obtained by prescribing the volume fraction of perovskite $X_{p v}$ in the assemblage, and by performing an appropriate average between the individual conductivities of perovskite and magnesio-wüstite, $\sigma_{p v}$ and $\sigma_{m w}$, as calculated using Eq. (2). Defining an appropriate average scheme to determine the electrical conductivity of a multiphase system is a sensitive issue, in particular because the individual conductivities of each mineral may strongly differ (for instance, $\sigma_{p v}$ and $\sigma_{m w}$ typically differ by 1 or 2 orders of magnitude, depending on the temperature and iron fraction). Different average schemes may lead to very different estimates of aggregate conductivities. The latter should however yield within the lower and upper Hashin-Shriktman bounds (HS- and HS+), which are based on variational principles and are the narrowest possible bounds for a multiphase system (Hashin and Shriktman 1962). For an aggregate of perovskite and magnesio-wüstite, and noting that the conductivity of magnesio-wüstite is larger than that of perovskite, these bounds are

$\sigma_{\mathrm{HS}-}=\sigma_{p v}+\frac{\left(1-X_{p v}\right) \sigma_{p v}\left(\sigma_{m w}-\sigma_{p v}\right)}{\sigma_{p v}+X_{p v}\left(\sigma_{m w}-\sigma_{p v}\right) / 3}$
$\sigma_{\mathrm{HS}+}=\sigma_{m w}-\frac{X_{p v} \sigma_{m w}\left(\sigma_{m w}-\sigma_{p v}\right)}{\sigma_{m w}+\left(1-X_{p v}\right)\left(\sigma_{m w}-\sigma_{p v}\right) / 3}$

The Voigt-Reuss-Hill (VRH) average (Hill 1963), which is often used to derive the thermo-elastic properties of multiphase aggregates, is the mean of the arithmetic and harmonic averages,

$\sigma_{\mathrm{VRH}}=\frac{1}{2}\left[X_{p v} \sigma_{p v}+\left(1-X_{p v}\right) \sigma_{m w}+\frac{\sigma_{p v} \sigma_{m w}}{\left(1-X_{p v}\right) \sigma_{p v}+X_{p v} \sigma_{m w}}\right](7)$

and is usually found to be biased towards the arithmetic average, i.e., towards the upper Hashin-Shriktman bound. By contrast, the geometric average (GM),

$\sigma_{\mathrm{GM}}=\sigma_{p v}{ }^{X_{p v}} \sigma_{m w}{ }^{\left(1-X_{p v}\right)}$

is usually found to be biased towards the lower 
Hashin-Shriktman bound (e.g., Shankland and Duba 1990; Khan and Shankland 2012). The effective medium theory (EMT) developed by Landauer (1952) for a two-phase aggregate assumes that each individual grain is surrounded by a homogeneous medium with conductivity $\sigma_{\mathrm{EMT}}$. Assuming that the total polarization induced by all individual grains should cancel out implies that $\sigma_{\text {EMT }}$ satisfies a degree two polynomial, of which it is the positive solution. For an assemblage of perovskite and magnesio-wüstite the conductivity thus is given by

$\sigma_{\mathrm{EMT}}=\frac{1}{4}\left[b+\sqrt{b^{2}+8 \sigma_{p v} \sigma_{m w}}\right]$

with $b=\sigma_{p v}\left(3 X_{p v}-1\right)-\sigma_{m w}\left(3 X_{p v}-2\right)$.

Figure 2 compares the Hashin-Shtrikman bounds and the conductivity obtained with the three estimators discussed above for an aggregate composed of $80 \%$ perovskite and 20\% magnesio-wüstite using the data in Table 1. Also shown are the conductivity for pure perovskite and pure magnesio-wüstite for an iron fraction $X_{F e}=0.1$. As noted in previous studies (e.g., Shankland and Duba 1990; Khan and Shankland 2012), the VRH average is close to the Hashin-Shtrikman upper bound throughout the lower mantle. By contrast, both the GM and EMT averages are close to Hashin-Shtrikman lower bound. However, it is important to note that all three estimators remain within the HashinShtrikman bounds. An interesting property shown in Fig. 2 is that the size of the Hashin-Shtrikman interval depends on the iron partitioning. As more iron goes to magnesio-wüstite (decreasing $K_{F e}$ ), the difference between the individual conductivities of perovskite and magnesio-wüstite is larger, which in turn increases the difference between the lower and upper Hashin-Shtrikman bounds. Note that in the case $K_{F e}=0.5$, because the fraction of iron in perovskite is only 0.086 , the lower Hashin-Strikman bound is smaller than the conductivity for pure perovskite, which was calculated for a fraction of iron of 0.1 .

When investigating the influence of temperature and composition on the lower mantle conductivity, it is convenient to prescribe the potential temperature $T_{p}$, i.e., the temperature at surface pressure. In the Earth's mantle compression induces an adiabatic increase in temperature that must be added to the potential temperature. The adiabatic gradient is given by

$\left(\frac{d T}{d P}\right)_{S}=\frac{\gamma T}{K_{S}}$

where $\gamma$ is the Grüneisen parameter, and $K_{S}$ the adiabatic bulk modulus of the assemblage at temperature $T$ and pressure $P$. The Grüneisen parameter is calculated from its surface value $\gamma_{0}$ following

$\gamma=\gamma_{0}\left(\frac{\rho}{\rho_{0}}\right)^{q}$

where $q$ is a constant and $\rho$ and $\rho_{0}$ the densities of the assemblage at $(T, P)$ and at the surface, respectively. $K_{S}$ and $\rho$ can be calculated from their surface values using an appropriate equation of state modelling. Here we used data and methods similar to those in Deschamps and Trampert (2004) to determine these quantities and the associated adiabatic gradient. Figure 3 illustrates the influence of the adiabatic increase in temperature with depth for two potential temperatures. At the bottom of the mantle, the adiabatic temperature increase can be as high as half the potential temperature and accounting for this effect increases the conductivity by $50-60 \%$ compared to the uncorrected value.

\subsection{Influence of Temperature and Composition}

Electrical conductivity increases with increasing real temperature (i.e., accounting for the adiabatic increase) $T_{\text {real }}$
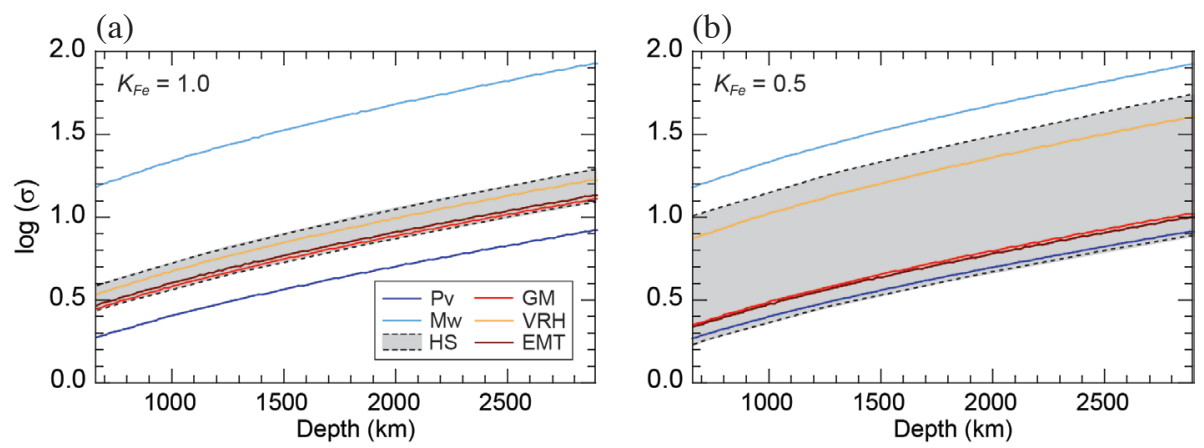

Fig. 2. Variation of electrical conductivity with depth for an assemblage of $80 \%$ perovskite and $20 \%$ magnesio-wüstite. The potential temperature is $T_{p}=2500 \mathrm{~K}$, the global iron fraction $X_{F e}=0.1$, and two values of the iron partitioning are considered, (a) $K_{F e}=1.0$, and (b) $K_{F e}=0.5$. The extent between the lower and upper Hashin-Shriktman bounds is denoted by the grey area, with three estimators calculated, geometric average (GM) (Eq. 8), Voigt-Reuss-Hill average (VRH) (Eq. 7), and effective medium theory (EMT) (Eq. 9). For compositions radial for pure perovskite (Pv) and pure magnesio-wüstite (Mw) are also shown (blue curves). 


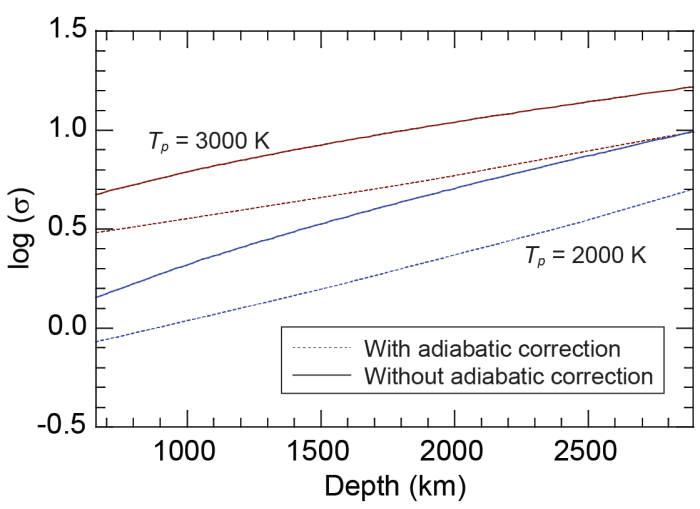

Fig. 3. Influence of the adiabatic increase of temperature with depth. The plain curves take the adiabatic contribution into account and the dotted curve neglect it. Two values of the potential temperature are considered, $T_{p}=2500 \mathrm{~K}$ (blue curves), and $T_{p}=3000 \mathrm{~K}$ (dark red curves). Other input parameters are $X_{F e}=0.1, X_{p v}=0.8$, and $K_{F e}=1.0$. Aggregate conductivities are calculated using the EMT average (Eq. 9).

throughout the mantle (Fig. 4a). The temperature effect is slightly attenuated with increasing depth. Figure 4a further indicates that a temperature excess of $500 \mathrm{~K}$ at the bottom of the mantle, as might be the case in LLSVP (Trampert et al. 2004; Deschamps et al. 2012) would result in a change in electrical conductivity between $20-30 \%$, depending on the average mantle temperature.

A careful examination at Table 1 indicates that magnesio-wüstite is electrically more conductive than perovskite by about two orders of magnitude. This holds throughout the lower mantle (blue curves in Fig. 2), as noted in previous studies (e.g., Vacher and Verhoeven 2007; Khan and Shankland 2012). Consequently, the mantle aggregate conductivity decreases with increasing perovskite fraction, $X_{p v}$. However, varying $X_{p v}$ triggers side effects making the detailed influence of this parameter more complex. Because the adiabatic gradient depends on the bulk modulus [Eq. (10)], $T_{\text {real }}$ indirectly depends on the compositional parameters, and in particular on $X_{p v}$. The bulk modulus of magnesiowüstite being smaller than that of perovskite, $T_{\text {real }}$ (and thus the conductivity of the aggregate) increases with decreasing perovskite fraction. This effect therefore enhances the purely compositional effect, i.e., the decrease in conductivity with increasing amount of perovskite. Figure $4 \mathrm{~b}$ plots the cumulated influence (chemical and differences in adiabatic corrections) of $X_{p v}$ on conductivity for $T_{\text {real }}=2500 \mathrm{~K}$, $X_{F e}=0.1$, and $K_{F e}=1.0$. For realistic mantle compositions, i.e., values of $X_{p v}$ in the range 0.6 - 0.9 , the conductivity calculated with EMT average varies between 1.5 - 12, depending on depth and on $X_{p v}$. However, the detailed influence of $X_{p v}$ further depends on the iron partitioning (Fig. 5). This complex dependence is due to the fact that the iron corrections are different for each mineral (parameters $\alpha$ and $\beta$ in Table 1), the conductivity of magnesio-wüstite being more sensitive to iron than that of perovskite. Because both $X_{p v}$ and $K_{F e}$ control the values of the individual iron fractions in perovskite and magnesio-wüstite [Eqs. (3) and (4)], changing the value of $K_{F e}$ modifies the effect of $X_{p v}$. For $K_{F e}=1.0$, the conductivity monotically decreases with increasing perovskite fraction, as shown in Fig. 5. By contrast, in the case $K_{F e}=0.3$, in which iron mostly goes to magnesio-wüstite, the influence of $X_{p v}$ is more complicated and is further affected by the average scheme used to determine the aggregate conductivity (blue curves in Fig. 5).

Figure $4 \mathrm{c}$ indicates that electrical conductivity increases with increasing iron fraction throughout the lower mantle. In the lowermost mantle $(z \geq 2500 \mathrm{~km})$, and for $X_{p v}=0.8$ and $K_{F e}=1.0$, an increase in $X_{F e}$ by 0.03 (i.e., $3.0 \%$ ) induces a threefold increase in conductivity. Unlike the effect of the perovskite fraction, the influence of the iron fraction does not substantially depend on the iron partitioning. For a given value $K_{F e}$, the electrical conductivity increases with increasing iron content, whatever the chosen value of $K_{F e}$.

As pointed out above, the detailed effect of iron partitioning depends on the volume fraction of perovskite. Figure 5 shows that it further depends on the average scheme used to calculate the aggregate conductivity. For values of $X_{p v}$ in the $0.6-0.9$ range, which are relevant to the Earth's lower mantle, electrical conductivity calculated with EMT average increases with increasing $K_{F e}$ (Fig. 4d), i.e., as more and more iron goes to perovskite. However, if a VRH average is used, the opposite is true, i.e., electrical conductivity decreases with increasing $K_{F e}$ (dotted curves in Fig. 5). Again, for values of $X_{p v}$ lower than 0.6, more complicated effects appear.

The results from the systematic search displayed in Figs. 4 and 5 have interesting implications for the lower mantle. Several models based on seismic tomography and the thermo-elastic data set have indicated that compared to the surrounding mantle, the LLSVP are hotter by about $500 \mathrm{~K}$ and enriched in iron and perovskite by about 3.0 and $10 \%$, respectively (Trampert et al. 2004; Deschamps et al. 2012; Mosca et al. 2012). According to Fig. 4, the excesses in temperature and iron fraction observed in LLSVP should both result in an increase in conductivity. The implications of variations in the perovskite fraction are more difficult to assess because they depend on the iron partitioning and on the average scheme (Fig. 5). It should be pointed out, however, that this effect is moderate, compared to that of iron. The aggregate conductivity changes by only $20 \%$ for a $10 \%$ excess of perovskite, but is multiplied by a factor three for a $3 \%$ increase in global iron fraction. Overall, LLSVP should appear as electrically more conductive than the surrounding mantle.

\section{ELECTRICAL CONDUCTIVITY FROM THERMO-CHEMICAL STRUCTURE}

Using the approach underlined in section 3 it is possible to determine synthetic horizontally averaged profiles 
(a)

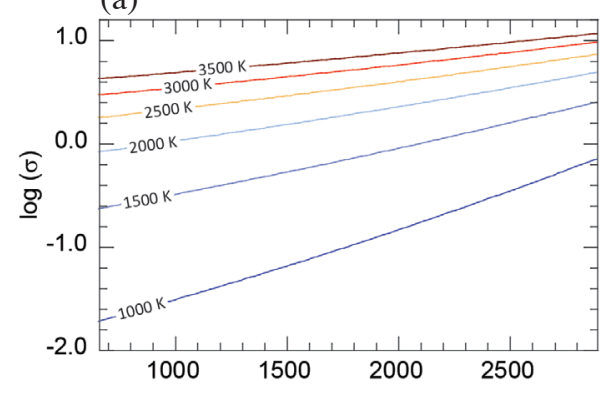

(c)

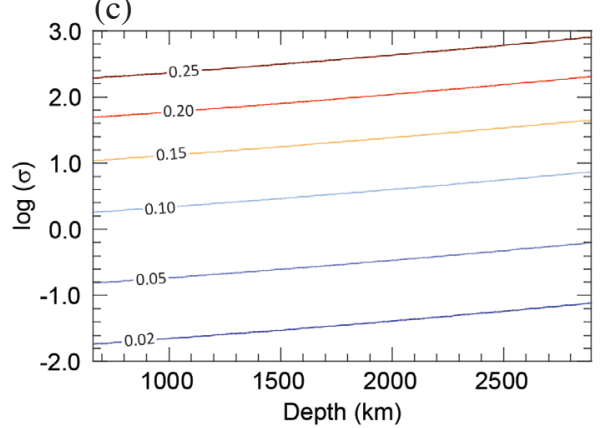

(b)

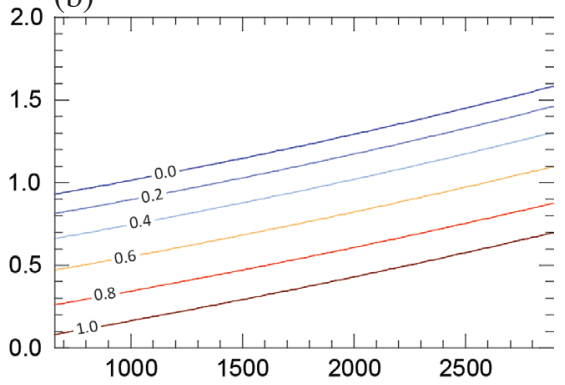

(d)

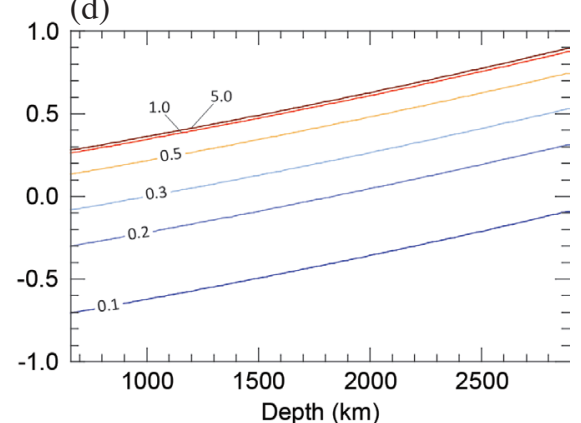

Fig. 4. Influence of temperature and composition on electrical conductivity. Results are plotted as a function of depth, and default real temperature, fraction of perovskite, global fraction of iron, and iron partitioning are $T_{\text {real }}=2500 \mathrm{~K}, X_{p v}=0.80, X_{F e}=0.1$, and $K_{F e}=1.0$, respectively. Average scheme is EMT. (a) Influence of the real temperature (see labels on curves). (b) Influence of the fraction of perovskite (see labels on curves). (c) Influence of the global fraction of iron (see labels on curves). (d) Influence of iron partitioning (see labels on curves).

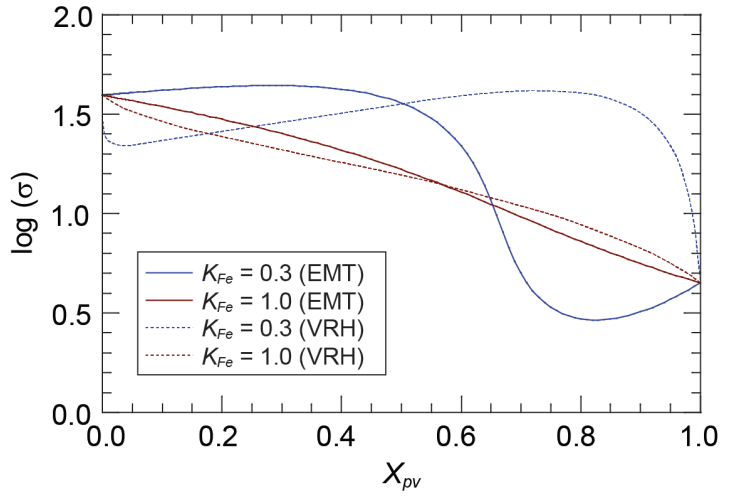

Fig. 5. Combined effects of the fraction of perovskite, $X_{p v}$, and of the iron partitioning, $K_{F e}$. The real temperature and global fraction of iron are $T_{\text {real }}=3000 \mathrm{~K}$ and $X_{F e}=0.1$, respectively. Calculations are made at depth $z=2500 \mathrm{~km}$, and two average schemes are considered, VRH and EMT.

and 3D distributions of lower mantle electrical conductivity from appropriate thermo-chemical models.

\subsection{Radial 1D Model}

The first application is designed to determine a 1D electrical conductivity radial model from the average thermochemical model of the Earth's mantle. The average thermochemical model used here is model DT2 from Deschamps and Trampert (2004). It is based on a Monte-Carlo search that takes into account uncertainties in the thermo-elastic parameters of mantle minerals, and derives a horizontally average (or reference) thermo-chemical model from all models that fit PREM seismic velocities and density (Dziewonski and Anderson 1981) within 1\%. In model DT2 the reference real (i.e., including the adiabatic gradient) temperature ranges from $2000 \mathrm{~K}$ at the top of the lower mantle to $2500 \mathrm{~K}$ at its base, with error bars (taken as one standard deviation of a nearly Gaussian distribution) of about $500 \mathrm{~K}$. The reference perovskite fraction varies between $0.7 \pm 0.1$ and $0.8 \pm 0.1$, depending on the depth and the reference iron global fraction is nearly constant with depth, around $0.09 \pm 0.02$. At each depth the individual perovskite and magnesio-wüstite conductivities are calculated with Eq. (2). Hasin-Shtrikman bounds [Eqs. (5) and (6)] and three different estimators \{GM [Eq. (8)], VRH [Eq. (7)], and EMT [Eq. (9)]\}, of the aggregate conductivity are then calculated. Uncertainties in the radial thermo-chemical model and in mineral physics data (Table 1) are taken into account using a Monte-Carlo search. The iron partitioning [Eq. (3)] is randomly varied in the 0.2 - 1.0 range. A total of $10^{4}$ reference thermo-chemical models and $10^{5}$ combinations of the electrical conductivity parameters lead to a collection of radial conductivity models, from which a mean and a standard deviation are calculated.

The resulting radial electrical conductivity model is plotted in Fig. 6, together with the models observed by Kuvshinov and Olsen (2006) and Velímský (2010). Observed models fit well within the Hashin-Shtrikman bounds except 
at the very top of the lower mantle $(670-800 \mathrm{~km})$. The conductivity calculated from EMT average ranges from $0.6 \mathrm{~S} \mathrm{~m}^{-1}$ $[\log (\sigma)=0.25]$ at the top of the lower mantle to about $16 \mathrm{~S} \mathrm{~m}^{-1}$ $[\log (\sigma)=1.2]$ at its base. Interestingly, it fits well the model observed by Velímský (2010) in the 800 - 1000 km layer and in the lowermost mantle (2200 - $2891 \mathrm{~km})$. The conductivity profile predicted by GM average remains close to the lower Hashin-Shtrikman bound throughout the lower mantle. By contrast the conductivity obtained from VRH average is close to the upper Hashin-Shtrikman bound and varies from $8 \mathrm{~S} \mathrm{~m}^{-1}[\log (\sigma)=0.9]$ at the top to about $60 \mathrm{~S} \mathrm{~m}^{-1}$ $[\log (\sigma)=1.8]$ at the bottom, which is always larger than the values observed by both Kuvshinov and Olsen (2006) and Velímský (2010). Note that in all cases, error bars on $\log (\sigma)$ (defined as one standard deviation around the mean) is large, typically around 0.5 .

\subsection{D Structure}

Mantle electrical conductivity maps may be recovered from the thermo-chemical structure inferred by probabilistic tomography (Trampert et al. 2004) (Fig. 1). These thermochemical distributions are varied within their error bars and added to the 1D radial thermo-chemical model DT2 (Deschamps and Trampert 2004), which is used as the reference model. At each point in the model the perovskite and magnesio-wüstite electrical conductivities are deduced from Eq. (2), and three estimators of the aggregate conductivity are calculated. Again, mineral physics data are varied within their error bars, generating a collection of conductivity maps, from which the mean and standard deviation are calculated. Figures 7 - 9 plot the resulting conductivity map (logarithm and relative anomalies), for the three layers defined by probabilistic tomography. Table 2 lists the average, minimum and maximum in these distributions for all three estimators and Hashin-Shtrikman bounds. It should be pointed out, however, that the RMS in the error bars (taken as one standard deviation around the mean value) is large, around 0.6 on $\log (\sigma)$.

A striking feature appearing in the lowermost mantle $(2000-2891 \mathrm{~km})$ is a belt of high electrical conductivity running along the equator (Fig. 9), and peaking in the LLSVP. Interestingly, this belt is predicted by all three estimators. According to EMT average, conductivity in the layer $2000-2891 \mathrm{~km}$ is up to $12 \mathrm{~S} \mathrm{~m}^{-1}$ (Table 2), leading to relative anomalies around $90 \%$, compared to the average conductivity. The maximum conductivity predicted by VRH average is around $20 \mathrm{~S} \mathrm{~m}^{-1}$, and relative anomalies are up to $97 \%$. In the mid lower mantle $(1200-2000 \mathrm{~km})$, the mean conductivity obtained with EMT and VRH average schemes are 1.7 and $4.4 \mathrm{~S} \mathrm{~m}^{-1}$, respectively, and lateral relative anomalies are moderate, up to $30-40 \%$ (Fig. 8). Finally, in the upper part of the lower mantle $(670-1200 \mathrm{~km})$, the mean electrical conductivity is comparable to that in the middle mantle (around 1.6 and $3.4 \mathrm{~S} \mathrm{~m}^{-1}$ for EMT and VRH averages, respectively), but relative anomalies are strong again, around $80 \%$ or more, depending on the average scheme, the largest anomalies being observed beneath the north-eastern Pacific, South Atlantic, and Indian Oceans.

Global models of lower mantle electrical conductivity based on magnetic field variations with period ranging from a few to 100 days have recently been obtained (Kelbert et al. 2009; Semenov and Kuvshinov 2012). The models of Kelbert et al. (2009) (KSE09) and Semenov and Kuvshinov (2012) (SK12) extend down to 2000 and $1600 \mathrm{~km}$, respectively. Lack of data and strong trade-off in the interpretation of long period ( $>1$ year) variations of the magnetic field, do not yet allow inferring electrical conductivity distributions at larger depths. Due to differences in the data used and inversion methods, KSE09 and SK12 are substantially different. In particular, the conductivity from SK12 is on average larger than that of KSE09 by about a factor of 2 and the correlation between the two models is low $(0.183,-0.190$, and 0.079 in the layers 670 - 900, $900-1200$, and $1200-1600 \mathrm{~km}$, respectively). Furthermore, relative lateral variations in conductivity are more pronounced in KSE09 than in SK12.

Comparing electrical conductivity maps inferred from the lower mantle thermo-chemical structure with the observed values raises several difficulties. First, spatial filtering of the observed models is required according to the vertical parameterization and lateral resolution of the thermo-chemical distributions used to infer the modeled conductivity. A potentially more serious problem is related to the frequency

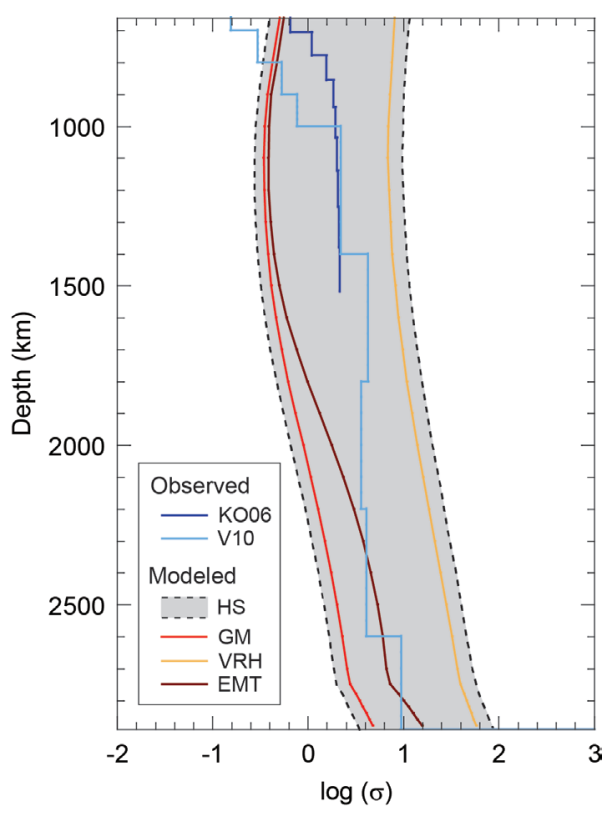

Fig. 6. Observed and modelled radial models of electrical conductivity. Three estimators are shown, GM, VRH, and EMT. The grey area extends from the lower to the upper Hashin-Shriktman bound. Observed models are from Kuvshinov and Olsen (2006) (KO06), and Velímský (2010) (V10). 

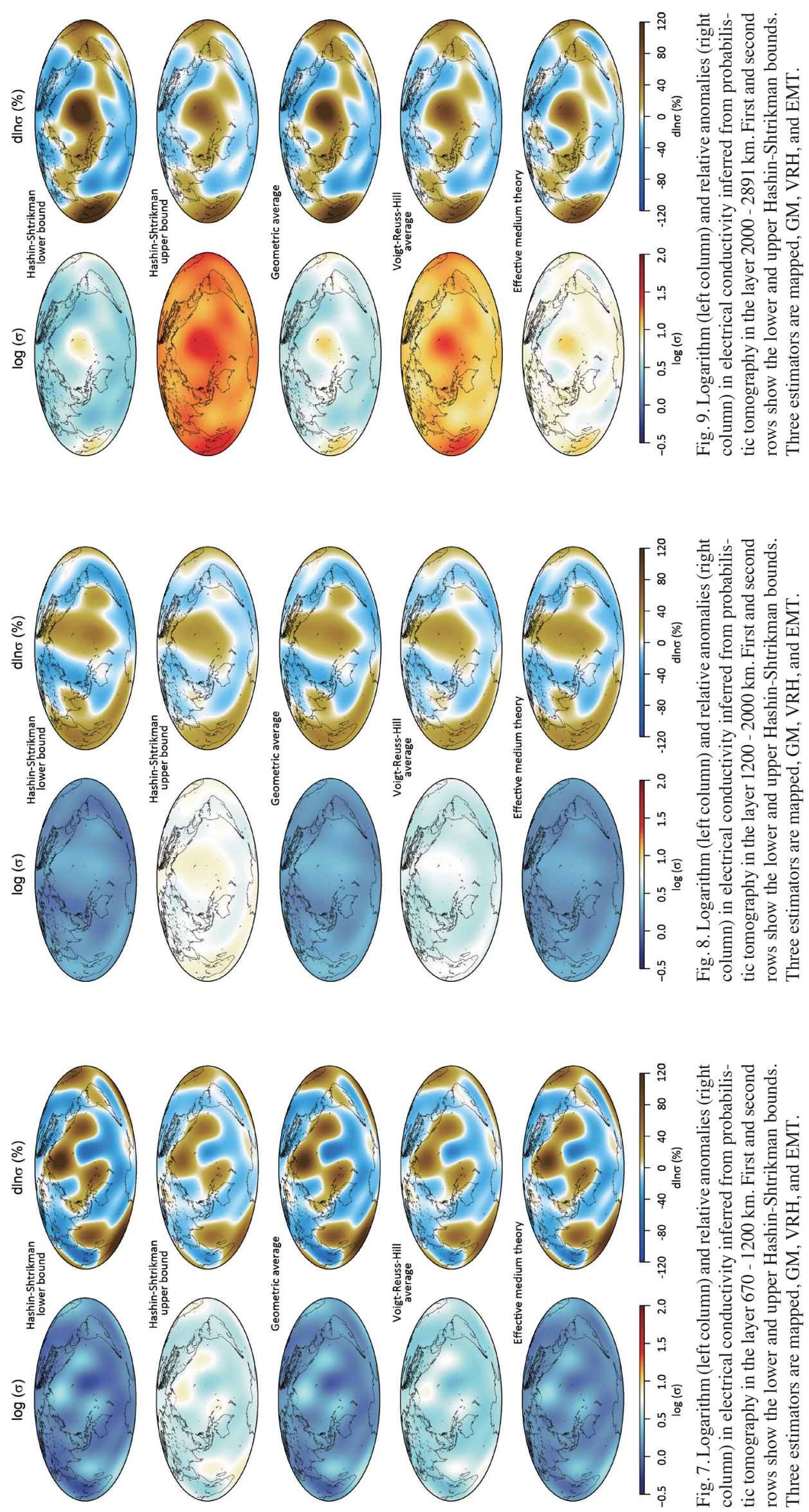
Table 2. Some statistics on modeled electrical conductivity.

\begin{tabular}{c|c|rcc|ccc}
\hline \multirow{2}{*}{ Layer (km) } & \multirow{2}{*}{ Average scheme } & \multicolumn{3}{|c|}{$\log (\boldsymbol{\sigma})$} & \multicolumn{3}{c}{$\boldsymbol{\delta} \boldsymbol{\sigma}(\boldsymbol{\%})$} \\
\cline { 2 - 7 } & & mean & $\min$ & $\max$ & RMS & min & max \\
\hline \multirow{3}{*}{$670-1200$} & HS- & 0.12 & -0.19 & 0.54 & 34.7 & -53.2 & 149.1 \\
& HS+ & 0.64 & 0.47 & 0.87 & 18.8 & -34.2 & 67.0 \\
& GM & 0.17 & -0.11 & 0.56 & 31.5 & -48.9 & 134.6 \\
& VRH & 0.53 & 0.33 & 0.79 & 21.2 & -37.5 & 78.9 \\
& EMT & 0.21 & 0.01 & 0.56 & 27.3 & -40.0 & 119.3 \\
\hline \multirow{2}{*}{$1200-2000$} & HS- & 0.16 & 0.01 & 0.34 & 19.0 & -29.8 & 50.5 \\
& HS+ & 0.76 & 0.66 & 0.88 & 11.7 & -19.8 & 32.8 \\
& GM & 0.21 & 0.09 & 0.38 & 17.2 & -26.0 & 43.5 \\
& VRH & 0.64 & 0.53 & 0.77 & 12.5 & -22.0 & 33.8 \\
& EMT & 0.23 & 0.14 & 0.38 & 15.2 & -21.9 & 36.2 \\
\hline \multirow{2}{*}{$2000-2891$} & HS- & 0.51 & 0.33 & 0.96 & 35.0 & -37.4 & 167.1 \\
& HS+ & 1.20 & 1.08 & 1.46 & 19.2 & -23.9 & 80.0 \\
& GM & 0.62 & 0.45 & 1.02 & 29.2 & -35.5 & 139.6 \\
& VRH & 1.06 & 0.93 & 1.34 & 21.4 & -26.0 & 90.4 \\
& EMT & 0.78 & 0.60 & 1.08 & 19.9 & -34.9 & 97.5 \\
\hline
\end{tabular}

Note: Listed values are the mean, minimum and maximum in the logarithm of electrical conductivity and the RMS, minimum and maximum in the relative anomalies in electrical conductivity. Average scheme are lower and upper Hashin-Shtrikman bounds (HS- and HS+), GM, VRH, and EMT.

of the geomagnetic signal used to build KSE09 and SK12, which may be inconsistent with the conductivity deduced from the thermo-chemical structure. Due to skin depth effects, only magnetic field variations with frequencies lower than a limit value may be observed at the surface of the Earth. The limit frequency decreases with increasing mantle conductivity. Therefore, large values of the lowermost mantle conductivity may decrease the limit frequency such that part of the geomagnetic signals used to build KSE09 and SK12 may have higher frequencies. Careful comparisons between modeled and observed conductivities would then require estimating the limit frequency imposed by the modeled conductivity, and filtering the observed conductivity maps according to this estimated limit frequency.

Figure 10 compares the modeled conductivity with spatially filtered models KSE09 and SK12. Conductivities from KSE09 and SK12 were averaged in layer 670 - 1200 and $1200-2000 \mathrm{~km}$, and filtered for spherical harmonic degrees 2, 4, and 6. Note that SK12 is limited to $1600 \mathrm{~km}$ depth and that the structure in the layer $1200-2000 \mathrm{~km}$ was assumed to be similar to that in the original SK12 layer $1200-1600 \mathrm{~km}$. Modeled conductivity calculated with EMT average is comparable in amplitude to that observed by SK12, but its distribution correlates very poorly with that of SK12. Conductivity obtained with VRH average is larger by a factor of 2 compared to SK12. Disagreement between the modeled conductivities and that mapped by KSE09 is more pronounced, both in amplitude and lateral variations.
However, taking uncertainties in the modeled conductivities into account, the synthetic conductivity inferred from thermo-chemical structure is not totally inconsistent with the conductivity observed in KSE09 and SK12.

\section{CONCLUDING DISCUSSION}

This study attempted to infer the electrical conductivity of the Earth's mantle from its thermo-chemical structure. The resulting radial conductivity model increases from $0.6 \mathrm{~S} \mathrm{~m}^{-1}$ at the top of the lower mantle, to $16 \mathrm{~S} \mathrm{~m}^{-1}$ at its base. Lateral variations in conductivity are strong at the top $(670-1200 \mathrm{~km})$ of the lower mantle and at its bottom (2000 - $2891 \mathrm{~km})$, and moderate in the intermediate layer $(1200-2000 \mathrm{~km})$. Certainly the most interesting feature, which needs to be further investigated in the future, is the presence of a high conductivity belt at the bottom of the mantle along the equator. This structure is present whatever the average scheme used to estimate the aggregate conductivity and it results from the fact that the low shear-wave velocity provinces observed by seismic tomography are enriched in iron and, at least part of them, hotter than the surrounding mantle (Fig. 1). Several directions may be followed in future works to improve the models discussed in this study.

The conductivity models calculated in this study have large uncertainties and their agreement with the global tomographic models of electrical conductivity KSE09 (Kelbert et al. 2009) and SK 12 (Semenov and Kuvshinov 2012) is poor. 

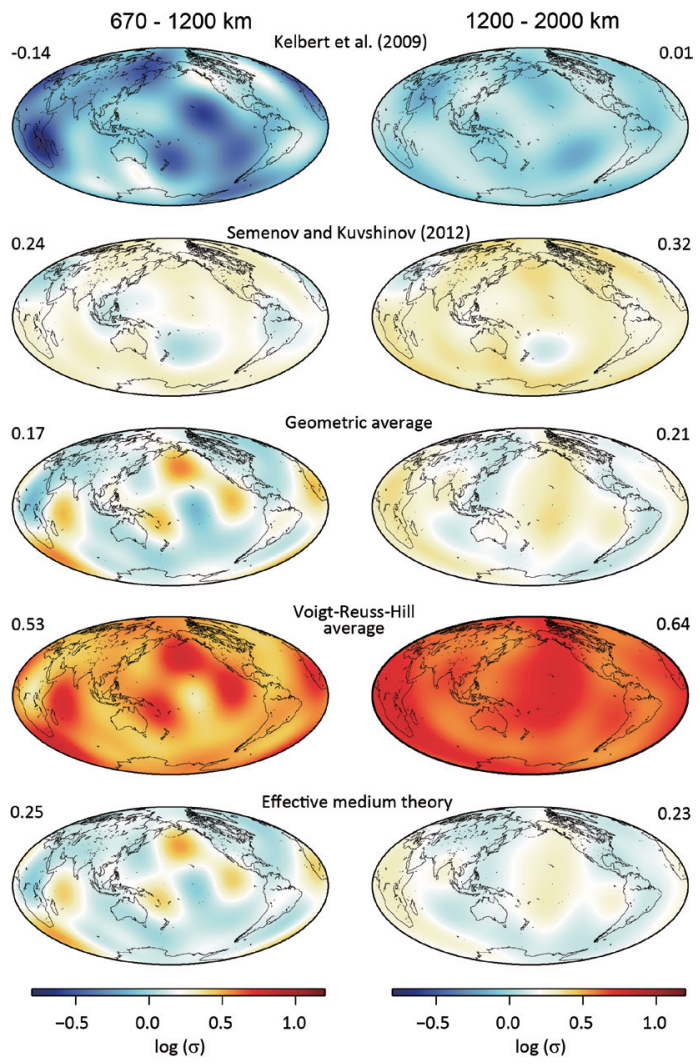

Fig. 10. Comparison between observed and modelled electrical conductivity in the layer $670-1200 \mathrm{~km}$ (left column) and $1200-2000 \mathrm{~km}$ (right column). Observed electrical conductivity are from Kelbert et al. (2009) and Semenov and Kuvshinov (2012), and are filtered vertically and laterally for spherical harmonic degrees 2,4 , and 6 . Three estimators are mapped, GM, VRH, and EMT.

Large uncertainties are directly related to uncertainties in the mineral physics data (Table 1) and in thermo-chemical models of the Earth's mantle. These latter further result from uncertainties in thermo-elastic data and in the reference thermo-chemical radial model. A better knowledge of mantle mineral and aggregate thermo-elastic and electrical properties is therefore needed to obtain a finer estimate of the electrical conductivity from the thermo-chemical structure. It should be kept in mind that due to differences in the data set and inversion methods, the conductivities mapped by KSE09 and SK12 strongly disagree. The discrepancies between the modeled conductivity and the observed models are comparable to the discrepancies that exist between KSE09 and SK12. Electrical conductivity tomographic maps may lack resolution at lower mantle depths. Finally, as pointed out in section 4, the geomagnetic signals used to build KSE09 and SK12 include signals with frequencies that may be incompatible with the conductivity deduced from the mantle thermo-chemical structure. More accurate comparisons would thus require a frequency filtering of the observed electrical conductivity maps.

The chemical anomalies inferred from probabilistic to- mography were parameterized in terms of iron and silicate (perovskite) anomalies. Clearly, other chemical heterogeneities may be present in the deep mantle, including recycled oceanic crust (MORB). Slabs may temporarily stack below the 670 phase transition (Kárason and van der Hilst 2000; Fukao et al. 2001), in which case they may partially explain the seismic anomalies observed at these depths. MORB may also reach the bottom of the mantle and stack there, as suggested by thermo-chemical convection models (e.g., Nakagawa et al. 2010). Unless they are very hot, recycled MORB are unlikely to explain LLSVP (Deschamps et al. 2012). They may however be present in local pools or even incorporated in small amounts into LLSVP (Tackley 2012; Li et al. 2014). Compared to pyrolite, MORB are enriched in iron and free of perovskite. All other effects being equal, they would therefore be more conductive than pyrolitic mantle. Recent experiments (Ohta et al. 2010) suggested that the electrical conductivity of MORB strongly increases with pressure. According to these experiments the conductivity of MORB at $51 \mathrm{GPa}(1200 \mathrm{~km}$-depth) varies between $3-10 \mathrm{~S} \mathrm{~m}^{-1}$ for temperatures in the $1400-2100 \mathrm{~K}$ range. Such values are comparable to those obtained for a pyrolitic composition. Slab stacking at the top of the lower mantle $(670-1000 \mathrm{~km})$ may thus significantly influence the lateral variations in electrical conductivity at these depths. To provide better electrical conductivity estimates, mantle thermochemical models should therefore include recycled oceanic crust. At lowermost mantle pressure, the experiments of Ohta et al. (2010) suggested that MORB are much more conductive that pyrolite, with values around $300 \mathrm{~S} \mathrm{~m}^{-1}$ at $133 \mathrm{GPa}$ and $2500 \mathrm{~K}$. This is about one order of magnitude larger than the conductivity inferred from iron and silicate rich material. Furthermore, it has been proposed that the electrical conductivity of the post-perovskite phase is also large, with values around $50 \mathrm{~S} \mathrm{~m}^{-1}$ at $129 \mathrm{GPa}$ and $2500 \mathrm{~K}$ (Ohta et al. 2008). The presence of high pressure MORB and post-perovskite in the lowermost mantle may thus result in a layer of large electrical conductivity at these depths, with values about one order of magnitude larger than the conductivity reconstructed from probabilistic tomography. It should be pointed out, however, that available radial conductivity models do not report any strong increase in conductivity in the deep mantle (Fig. 6). Mantle electrical conductivity remains around $4.0 \mathrm{~S} \mathrm{~m}^{-1}$ from $1000-2600 \mathrm{~km}$ depth and slightly increases to $10 \mathrm{~S} \mathrm{~m}^{-1}$ at greater depths (Velímský 2010).

Three dimensional images of electrical conductivity in the lowermost mantle are needed to discriminate between the possible thermo-chemical models and to determine the relative amount of each component (e.g., recycled MORB, post-perovskite, reservoirs of primordial material enriched in iron). So far the global electrical conductivity tomographic models (Kelbert et al. 2009; Semenov and Kuvshinov 2012) could not image regions deeper than $2000 \mathrm{~km}$, in particular 
because this would require longer period (1 year and larger) geomagnetic signals, which are still sparse. In addition, at these periods the variations in magnetic field may have several origins (including sources from the core and from the magnetosphere), which are difficult to separate from the magnetic field induced by lower mantle electrical currents. Additional data and methodological developments are therefore needed to map electrical conductivity in this region.

Acknowledgements The author would like to thank two anonymous colleagues for their detailed and useful comments that greatly helped to improve a first version of this article. The research presented in this paper was funded by the National Science Council of Taiwan (NSC) grant 101-2116-M-001-001-MY3, and by Academia Sinica grant AS-102-CDA-M02.

\section{REFERENCES}

Banks, R. J., 1969: Geomagnetic variations and the electrical conductivity of the upper mantle. Geophys. $J$. Int., 17, 457-487, doi: 10.1111/j.1365-246X.1969. tb00252.x. [Link]

Connolly, J. A. D., 2005: Computation of phase equilibria by linear programming: A tool for geodynamic modeling and its application to subduction zone decarbonation. Earth Planet. Sci. Lett., 236, 524-541, doi: 10.1016/j. eps1.2005.04.033. [Link]

Deschamps, F. and J. Trampert, 2004: Towards a lower mantle reference temperature and composition. Earth Planet. Sci. Lett., 222, 161-175, doi: 10.1016/j. eps1.2004.02.024. [Link]

Deschamps, F., L. Cobden, and P. J. Tackley, 2012: The primitive nature of large low shear-wave velocity provinces. Earth Planet. Sci. Lett., 349-350, 198-208, doi: 10.1016/j.epsl.2012.07.012. [Link]

Dobson, D. P. and J. P. Brodholt, 2000: The electrical conductivity of the lower mantle phase magnesiowüstite at high temperatures and pressures. J. Geophys. Res., 105, 531-538, doi: 10.1029/1999JB900242. [Link]

Dziewonski, A. M. and D. L. Anderson, 1981: Preliminary reference Earth model. Phys. Earth Planet. Inter., 25, 297-356, doi: 10.1016/0031-9201(81)90046-7. [Link]

Fukao, Y., S. Widiyantoro, and M. Obayashi, 2001: Stagnant slabs in the upper and lower mantle transition region. Rev. Geophys., 39, 291-323, doi: 10.1029/1999RG000068. [Link]

Goddat, A., J. Peyronneau, and J. P. Poirier, 1999: Dependence on pressure of conduction by hopping of small polarons in minerals of the Earth's lower mantle. Phys. Chem.Miner., 27, 81-87, doi: 10.1007/s002690050243. [Link]

Hashin, Z. and S. Shtrikman, 1962: A variational approach to the theory of the effective magnetic permeability of multiphase materials. J. Appl. Phys., 33, 3125-3131, doi: 10.1063/1.1728579. [Link]

Hill, R., 1963: Elastic properties of reinforced solids: Some theoretical principles. J. Mech. Phys. Solids, 11, 357372, doi: 10.1016/0022-5096(63)90036-X. [Link]

Houser, C., G. Masters, P. Shearer, and G. Laske, 2008: Shear and compressional velocity models of the mantle from cluster analysis of long-period waveforms. Geophys. J. Int., 174, 195-212, doi: 10.1111/j.1365-246X.2008.03763.x. [Link]

Ishii, M. and J. Tromp, 1999: Normal-mode and free-air gravity constraints on lateral variations in velocity and density of Earth's mantle. Science, 285, 1231-1236, doi: 10.1126/science.285.5431.1231. [Link]

Javoy, M., E. Kaminski, F. Guyot, D. Andrault, C. Sanloup, M. Moreira, S. Labrosse, A. Jambon, P. Agrinier, A. Davaille, and C. Jaupart, 2010: The chemical composition of the Earth: Enstatite chondrite models. Earth Planet. Sci. Lett., 293, 259-268, doi: 10.1016/j. eps1.2010.02.033. [Link]

Kárason, H. and R. D. van der Hilst, 2000: Constraints on mantle convection from seismic tomography. In: Richards, M. A., R. G. Gordon, and R. D. van der Hilst (Eds.), The History and Dynamics of Global Plate Motions, Geophysical Monograph Series, Vol. 121, American Geophysical Union, Washington, D.C., 277288, doi: 10.1029/GM121. [Link]

Katsura, T., K. Sato, and E. Ito, 1998: Electrical conductivity of silicate perovskite at lower-mantle conditions. Nature, 395, 493-495, doi: 10.1038/26736. [Link]

Kelbert, A., A. Schultz, and G. Egbert, 2009: Global electromagnetic induction constraints on transition-zone water content variations. Nature, 460, 1003-1007, doi: 10.1038/nature08257. [Link]

Khan, A. and T. J. Shankland, 2012: A geophysical perspective on mantle water content and melting: Inverting electromagnetic sounding data using laboratory-based electrical conductivity profiles. Earth Planet. Sci. Lett., 317-318, 27-43, doi: 10.1016/j.eps1.2011.11.031. [Link]

Kustowski, B., G. Ekström, and A. M. Dziewoński, 2008: Anisotropic shear-wave velocity structure of the Earth's mantle: A global model. J. Geophys. Res., 113, B06306, doi: 10.1029/2007JB005169. [Link]

Kuvshinov, A. and N. Olsen, 2006: A global model of mantle conductivity derived from 5 years of CHAMP, Ørsted, and SAC-C magnetic data. Geophys. Res. Lett., 33, L18301, doi: 10.1029/2006GL027083. [Link]

Kuvshinov, A. and A. Semenov, 2012: Global 3-D imaging of mantle electrical conductivity based on inversion of observatory $C$-responses-I. An approach and its verification. Geophys. J. Int., 189, 1335-1352, doi: 10.1111/j.1365-246X.2011.05349.x. [Link]

Landauer, R., 1952: The electrical resistance of binary 
metallic mixtures. J. Appl. Phys., 23, 779-784, doi: 10.1063/1.1702301. [Link]

Lee, C. T. A., P. Luffi, T. Höink, J. Li, R. Dasgupta, and J. Hernlund, 2010: Upside-down differentiation and generation of a 'primordial' lower mantle. Nature, 463, 930-935, doi: 10.1038/nature08824. [Link]

Li, M., A. K. McNamara, and E. J. Garnero, 2014: Chemical complexity of hotspots caused by cycling oceanic crust through mantle reservoirs. Nat. Geosci., 7, 366-370, doi: 10.1038/ngeo2120. [Link]

Li, X. and R. Jeanloz, 1991: Effect of iron content on the electrical conductivity of perovskite and magnesiowüstite assemblages at lower mantle conditions. J. Geophys. Res., 96, 6113-6120, doi: 10.1029/90JB02632. [Link]

Masters, G., G. Laske, H. Bolton, and A. Dziewonski, 2000: The relative behavior of shear velocity, bulk sound speed, and compressional velocity in the mantle: Implications for chemical and thermal structure. In: Karato, S. I., A. Forte, R. Liebermann, G. Masters, and L. Stixrude (Eds.), Earth's Deep Interior: Mineral Physics and Tomography From the Atomic to the Global Scale, Geophysical Monograph Series, Vol. 117, American Geophysical Union, Washington, D.C., 63-87, doi: 10.1029/GM117. [Link]

Mosca, I., L. Cobden, A. Deuss, J. Ritsema, and J. Trampert, 2012: Seismic and mineralogical structures of the lower mantle from probabilistic tomography. J. Geophys. Res., 117, B06304, doi: 10.1029/2011JB008851. [Link]

Nakagawa, T., P. J. Tackley, F. Deschamps, and J. A. D. Connolly, 2010: The influence of MORB and harzburgite composition on thermo-chemical mantle convection in a 3-D Spherical shell with self-consistently calculated mineral physics. Earth Planet. Sci. Lett., 296, 403-412, doi: 10.1016/j.eps1.2010.05.026. [Link]

Nomura, R., H. Ozawa, S. Tateno, K. Hirose, J. Hernlund, S. Muto, H. Ishii, and N. Hiraoka, 2011: Spin crossover and iron-rich silicate melt in the Earth's deep mantle. $\mathrm{Na}$ ture, 473, 199-203, doi: 10.1038/nature09940. [Link]

Ohta, K., S. Onoda, K. Hirose, R. Sinmyo, K. Shimizu, N. Sata, Y. Ohishi, and A. Yasuhara, 2008: The electrical conductivity of post-perovskite in Earth's D" Layer. Science, 320, 89-91, doi: 10.1126/science.1155148. [Link]

Ohta, K., K. Hirose, M. Ichiki, K. Shimizu, N. Sata, and Y. Ohishi, 2010: Electrical conductivities of pyrolitic mantle and MORB materials up to the lowermost mantle conditions. Earth Planet. Sci. Lett., 289, 497-502, doi: 10.1016/j.epsl.2009.11.042. [Link]

Olsen, N., 1999: Long-period (30 days-1 year) electromagnetic sounding and the electrical conductivity of the lower mantle beneath Europe. Geophys. J. Int., 138, 179-187, doi: 10.1046/j.1365-246x.1999.00854.x. [Link]

Poirier, J. P., 1991: Introduction to the Physics of the Earth's
Interior, Cambridge University Press, Cambridge, $264 \mathrm{pp}$.

Poirier, J. P. and J. Peyronneau, 1992: Experimental determination of the electrical conductivity of the material of the Earth's lower mantle. In: Syono, Y. and M. H. Manghnani (Eds.), High-Pressure Research: Application to Earth and Planetary Sciences, Geophysical Monograph Series, Vol. 67, American Geophysical Union, Washington, D.C., 77-87, doi: 10.1029/GM067. [Link]

Ritsema, J., A. Deuss, H. J. van Heijst, and J. H. Woodhouse, 2011: S40RTS: A degree-40 shear-velocity model for the mantle from new Rayleigh wave dispersion, teleseismic traveltime and normal-mode splitting function measurements. Geophys. J. Int., 184, 1223-1236, doi: 10.1111/j.1365-246X.2010.04884.x. [Link]

Semenov, A. and A. Kuvshinov, 2012: Global 3-D imaging of mantle conductivity based on inversion of observatory $C$-responses-II. Data analysis and results. Geophys. J. Int., 191, 965-992, doi: 10.1111/j.1365-246X.2012.05665.x. [Link]

Shankland, T. J. and A. G. Duba, 1990: Standard electrical conductivity of isotropic, homogeneous olivine in the temperature range $1200^{\circ}-1500^{\circ} \mathrm{C}$. Geophys. J. Int., 103, 25-31, doi: 10.1111/j.1365-246X.1990.tb01749.x. [Link]

Shankland, T. J., J. Peyronneau, and J. P. Poirier, 1993: Electrical conductivity of the Earth's lower mantle. Nature, 366, 453-455, doi: 10.1038/366453a0. [Link]

Shimizu, H., H. Utada, K. Baba, T. Koyama, M. Obayashi, and Y. Fukao, 2010: Three-dimensional imaging of electrical conductivity in the mantle transition zone beneath the North Pacific Ocean by a semi-global induction study. Phys. Earth Planet. Inter., 183, 252-269, doi: 10.1016/j.pepi.2010.01.010. [Link]

Tackley, P. J., 2012: Dynamics and evolution of the deep mantle resulting from thermal, chemical, phase and melting effects. Earth-Sci. Rev., 110, 1-25, doi: 10.1016/j.earscirev.2011.10.001. [Link]

Tarits, P. and M. Mandéa, 2010: The heterogeneous electrical conductivity structure of the lower mantle. Phys. Earth Planet. Inter., 183, 115-125, doi: 10.1016/j. pepi.2010.08.002. [Link]

Trampert, J., P. Vacher, and N. Vlaar, 2001: Sensitivities of seismic velocities to temperature, pressure and composition in the lower mantle. Phys. Earth Planet. Inter., 124, 255-267, doi: 10.1016/S0031-9201(01)00201-1. [Link]

Trampert, J., F. Deschamps, J. Resovsky, and D. Yuen, 2004: Probabilistic tomography maps chemical heterogeneities throughout the lower mantle. Science, 306, 853-856, doi: 10.1126/science.1101996. [Link]

Vacher, P. and O. Verhoeven, 2007: Modelling the electrical conductivity of iron-rich minerals for planetary applications. Planet. Space Sci., 55, 455-466, doi: 
10.1016/j.pss.2006.10.003. [Link]

Van der Hilst, R. D. and H. Kárason, 1999: Compositional heterogeneity in the bottom 1000 kilometers of Earth's mantle: Toward a hybrid convection model. Science, 283, 1885-1888, doi: 10.1126/science.283.5409.1885. [Link]

Van der Hilst, R. D., S. Widiyantoro, and E. R. Engdahl, 1997: Evidence for deep mantle circulation from global tomography. Nature, 386, 578-584, doi: 10.1038/386578a0. [Link]

Velímský, J., 2010: Electrical conductivity in the lower mantle: Constraints from CHAMP satellite data by timedomain EM induction modelling. Phys. Earth Planet. Inter., 180, 111-117, doi: 10.1016/j.pepi.2010.02.007.
[Link]

$\mathrm{Xu}, \mathrm{Y}$. and C. McCammon, 2002: Evidence for ionic conductivity in lower mantle $(\mathrm{Mg}, \mathrm{Fe})(\mathrm{Si}, \mathrm{Al}) \mathrm{O}_{3}$ perovskite. J. Geophys. Res., 107, 11-1-11-7, doi: 10.1029/2001JB000677. [Link]

Xu, Y., T. J. Shankland, and B. T. Poe, 2000: Laboratory-based electrical conductivity in the Earth's mantle. J. Geophys. Res., 105, 27865-27875, doi: 10.1029/2000JB900299. [Link]

Yoshino, T., T. Matsuzaki, A. Shatskiy, and T. Katsura, 2009: The effect of water on the electrical conductivity of olivine aggregates and its implications for the electrical structure of the upper mantle. Earth Planet. Sci. Lett., 288, 291-300, doi: 10.1016/j.eps1.2009.09.032. [Link] 